\title{
VLBI IMAGING OF THE GRAVITATIONAL LENSES B1422+231 AND MG J0414+0534
}

\author{
E. ROS \\ Max-Planck-Institut für Radioastronomie, Bonn, Germany \\ J.C. GUIRADO AND J.M. MARCAIDE \\ Dep. Astronomia i Astrofísica, U. València, Burjassot, Spain \\ M.A. PÉREZ-TORRES \\ Istituto di Radioastronomia-CNR, Bologna, Italy \\ E.E. FALCO \\ Harvard-Smiths. Center for Astrophysics, Cambridge MA, US \\ J.A. MUÑOZ \\ Instituto de Astrofísica de Canarias, La Laguna, Spain \\ AND \\ A. ALBERDI AND L. LARA \\ Instituto de Astrofísica de Andalucía/CSIC Granada, Spain
}

\begin{abstract}
We present wide-field images of the quadruple gravitational lenses B1422+231 and MG J0414+0534 obtained from global Very Long Baseline Interferometry (VLBI) observations at $8.4 \mathrm{GHz}$ on 23 November 1997. We present also a lens model for MG J0414+0534, which reproduces the core positions and flux densities of the VLBI images, combining a singular isothermal ellipsoid with external shear, and a singular isothermal sphere to represent, respectively, the main lens galaxy and its neighbor, a faint galaxy near one of the images.
\end{abstract}

Introduction. We have embarked on a project of multi-epoch observations of the quadruple images of the two gravitationally lensed objects B1422+231 and MG J0414+0534 to compare possible structural changes in the lensed images and measure possible shifts in the relative positions of the sub-images. Here we present our images from the first epoch observations on 23 November 1997. 


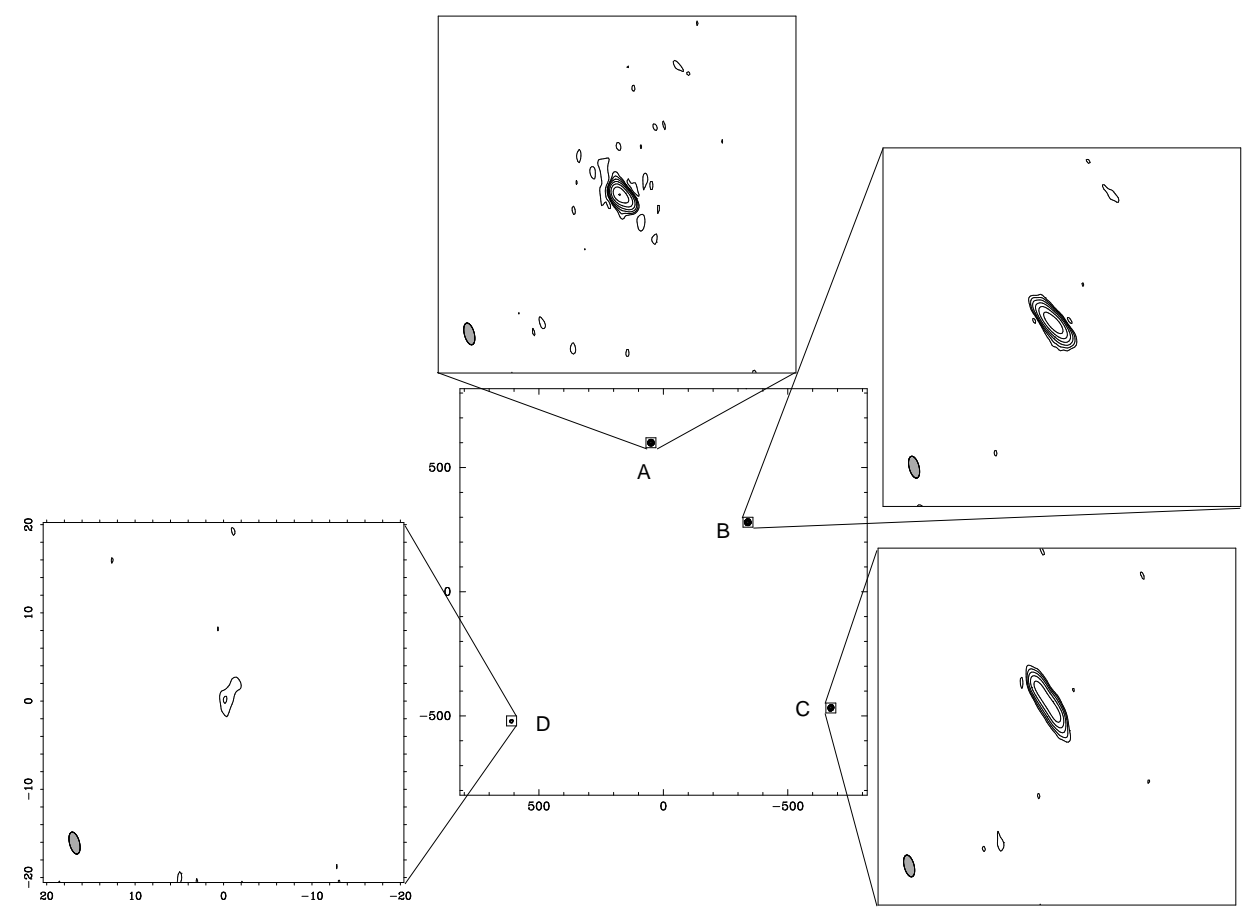

Figure 1. Composite of our preliminary maps of the four gravitationally lensed images of $\mathrm{B} 1422+231$ at $8.4 \mathrm{GHz}$, labeled as A (top, center), B (top, right), C (bottom, right), and D (bottom, left) with a global VLBI wide-field image (central panel) of the radio source. The axes are relative right ascension and declination, in milliarcseconds. The detailed figures are all convolved with a beam of $1.64 \times 1.16$ mas (position angle (P.A.) $22.43^{\circ}$ ), and the wide-field image is convolved with a circular 25 mas beam.

Imaging of B1422+231. B1422+231 is a quadruple gravitational lens object discovered by Patnaik et al. (1992). The maximum image separation between the four components is 1.3 arcsec. The background radio source is associated with a $15.5 \mathrm{mag}$ QSO at redshift $z=3.62$ (Patnaik et al. 1992). The lensing object is an elliptical galaxy at $z=0.338$ (Tonry 1998). The lensing system has been modelled with an elliptical potential and a shear (see Mao \& Schneider 1998 and references therein). The optical images from the Hubble Space Telescope (Impey et al. 1996) show the lens object close to the faintest sub-image. We show in Fig. 1 our VLBI images of $\mathrm{B} 1422+231$. The bright components, A, B, and C are stretched along the direction joining them, in accordance with (general) lens modeling. The much weaker image D shows some elongation towards the lensing galaxy. Our images are in good agreement with those obtained at $8.4 \mathrm{GHz}$ from Patnaik et al. (1999). The images are consistent with a background compact radio source without a prominent, extended jet-like structure. 


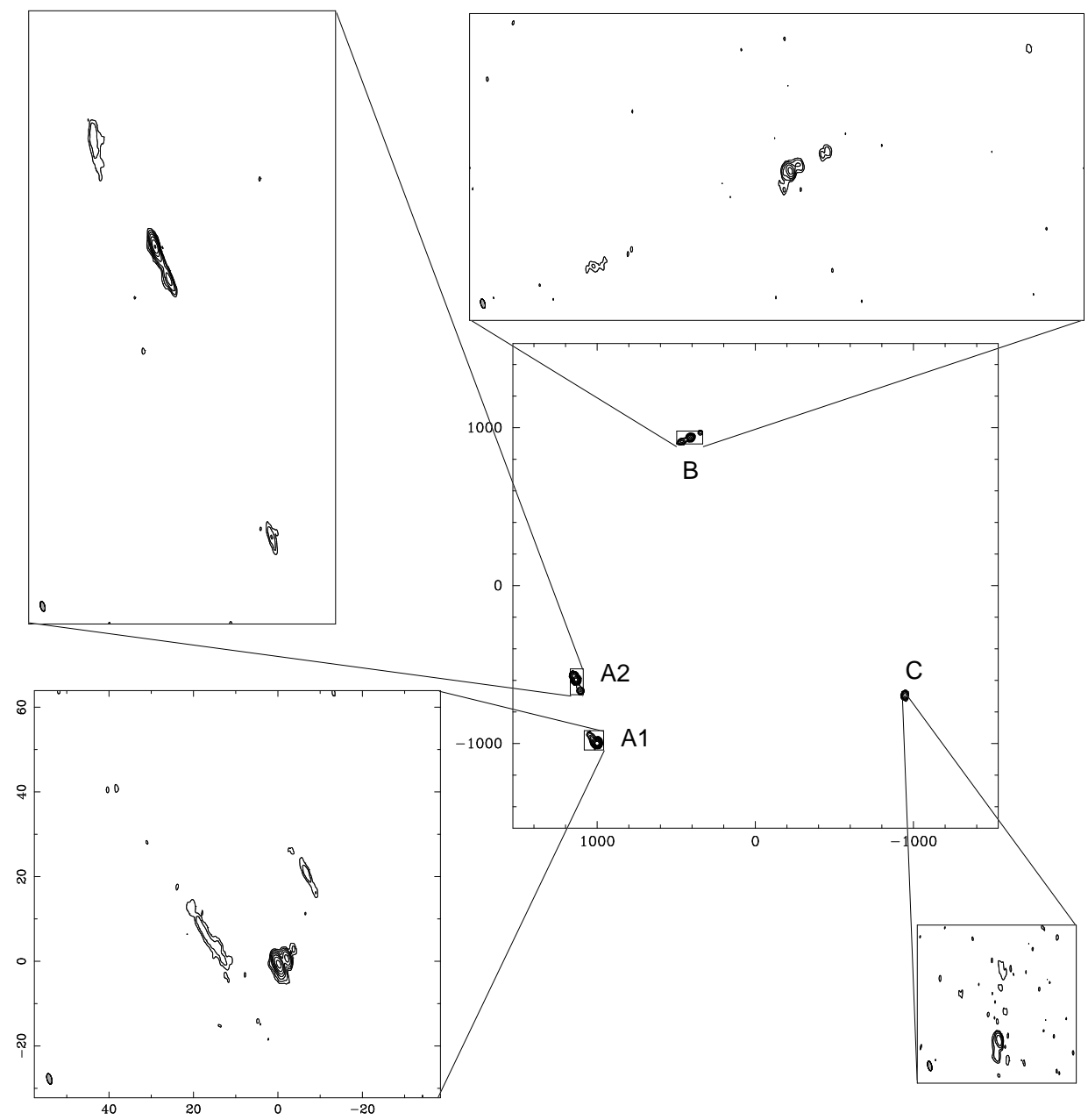

Figure 2. Composite of our images of the four gravitationally lensed images of MG J0414+0534, labeled as A1 (bottom, left), A2 (top, left), B (top, right), and C (bottom, right) at $8.4 \mathrm{GHz}$ with a global VLBI wide-field image (central panel) of the radio source. The axes are relative right ascension and declination, in milliarcseconds. The detailed images are convolved with a beam of $2.55 \times 1.13$ mas (P.A. $14.6^{\circ}$ ), and the wide-field image is convolved with a circular 25 mas beam. See also Ros et al. (2000).

Imaging and modeling of MGJ0414+0534. MG J0414+0534 is a quadruple gravitational lens system discovered by Hewitt et al. (1992), with maximum separations between components of 2.12 arcsec. The background object is a $21.2 \mathrm{mag}$ QSO with $z=2.64$ (Lawrence et al. 1995). The lensing potential is due to an elliptical galaxy at redshift $z=0.96$ (Tonry \& Kochanek 1999) and its likely neighbor, a galaxy $\sim 1.51$ arcsec away (P.A. of $-14^{\circ}$ ). Hubble Space Telescope observations revealed an optical blue arc joining the three brightest components (Falco et al. 1997). 
We present global VLBI images of this system in Fig. 2. The individual images exhibit radio structures extending up to 100 mas. The images are labeled, from the brightest to the weakest as A1, A2, B, and C. From lens modeling, the structure of $\mathrm{B}$ is the most similar to that of the background radio source, a double compact structure with a double jet. A1 shows the most complex structure, with the core-like region and both jets quite distorted. A2 is similar to $\mathrm{B}$, but rotated and mirrored. For $\mathrm{C}$ we only detect emission at the northern side of the jet. The morphology of our images is very similar to that presented in Trotter et al. (2000) at $5 \mathrm{GHz}$.

We reproduce successfully the relative positions and peak of brightness ratios of the radio cores with a lens model consisting of a singular isothermal ellipsoid (for the elliptical galaxy visible at the optical, and placed at the center between the four radio images) with an external shear, and a secondary potential given by a singular isothermal sphere (associated with a small object close to the west of component B). The model predicts that $\mathrm{B}$ is the leading image, and $\mathrm{C}$ lags all the other images. The delays from $\mathrm{B}$ are of $15.7 \pm 1.3,16.0 \pm 1.4$, and $66 \pm 5$ days for $\mathrm{A} 1, \mathrm{~A} 2$, and $\mathrm{C}$, respectively. In our model, the spherical isothermal ellipse potential (constrained by the images) is aligned with the optical images of the lens galaxy. We plan to refine and study further our lens model by adding the constraints provided by the lensed jets, using new software that avoids the problem of matching components along these jets, in the different lensed images.

We observed these radio sources again on 8 June 1999, and new observations are scheduled for late 2000. Once these new epochs are analyzed it might be possible to measure changes in the brightness peak positions of both radio sources and/or morphological evolution in the core-like and jet-like regions of MG J0414+0534.

Acknowledgements This work has been partially supported by the Spanish DGICYT Grants No. PB96-0782 and PB97-1164 and by European Comission's TMR-LSF programme, contract No. ERBFMGECT950012.

\section{References}

Falco E. E., Lehár J., Shapiro I. I., 1997, AJ 113, 540

Hewitt J. N., Turner E. L., Lawrence C. R., Schneider D. P., Brody J. P., 1992, AJ 104, 968

Impey C.D., Foltz C.B., Petry C.E., Browne I.W.A., Patnaik A.R., 1996, ApJ 462, L53

Lawrence C. R., Elston R., Jannuzi B. T., Turner E. L., 1995, AJ 110, 2570

Mao S., Schneider P., 1998, MNRAS 295, 587

Patnaik A. R., Browne, I. W. A., Walsh D., et al., 1992, MNRAS 259, 1P

Patnaik A.R., Kemball A.J., Porcas R.W., Garrett M.A., 1999, MNRAS 307, L1

Ros E., Guirado J. C., Marcaide J. M., et al., 2000, A\&A 362, 845

Tonry J. L., Kochanek C. S., 1999, AJ 117, 2034

Tonry J. L., 1998, AJ 115, 1

Trotter C. S., Winn J. N., Hewitt J. N., 2000, ApJ 535, 671 